\title{
Transfer of Pseudomonas plantarii and Pseudomonas glumae to Burkholderia as Burkholderia spp. and Description of Burkholderia vandii sp. nov.
}

\author{
TEIZI URAKAMI, ${ }^{*}$ CHIEKO ITO-YOSHIDA, ${ }^{2}$ HISAYA ARAKI, ${ }^{2}$ TOSHIO KIJIMA, ${ }^{3}$ \\ KEN-ICHIRO SUZUKI, ${ }^{4}$ AND KAZUO KOMAGATA ${ }^{5} \dagger$
}

Biochemicals Division, Mitsubishi Gas Chemical Co., Shibaura, Minato-ku, Tokyo 105, ${ }^{1}$ Niigata Research Laboratory, Mitsubishi Gas Chemical Co., Tayuhama, Niigata 950-31, ${ }^{2}$ Plant Pathological Division of Biotechnology,

Tochigi Agricultural Experiment Station, Utsunomiya 320, ${ }^{3}$ Japan Collection of Microorganisms, The Institute of Physical and Chemical Research, Wako-shi, Saitama 351-01, ${ }^{4}$ and Institute of Molecular Cell and Biology, The University of Tokyo, Bunkyo-ku, Tokyo $113,{ }^{5}$ Japan

\begin{abstract}
Plant-associated bacteria were characterized and are discussed in relation to authentic members of the genus Pseudomonas sensu stricto. Bacteria belonging to Pseudomonas rRNA group II are separated clearly from members of the genus Pseudomonas sensu stricto (Pseudomonas fluorescens rRNA group) on the basis of plant association characteristics, chemotaxonomic characteristics, DNA-DNA hybridization data, rRNA-DNA hybridization data, and the sequences of 5S and 16S rRNAs. The transfer of Pseudomonas cepacia, Pseudomonas mallei, Pseudomonas pseudomallei, Pseudomonas caryophylli, Pseudomonas gladioli, Pseudomonas pickettii, and Pseudomonas solanacearum to the new genus Burkholderia is supported; we also propose that Pseudomonas plantarii and Pseudomonas glumae should be transferred to the genus Burkholderia. Isolate VA-1316 ${ }^{\mathrm{T}}$ (T $=$ type strain) was distinguished from Burkholderia species on the basis of physiological characteristics and DNA-DNA hybridization data. A new species, Burkholderia vandii sp. nov. is proposed for this organism; the type strain of $B$. vandii is VA-1316 (= JCM 7957).
\end{abstract}

Palleroni et al. (32) reported in 1973 that Pseudomonas species could be divided into at least five distinct groups on the basis of levels of rRNA-DNA homology. This finding was supported by the variable enzymological patterns observed for tyrosine biosynthesis (6), cellular fatty acid composition data (27), hydroxy fatty acid composition data $(27,43)$, quinone system data $(27,61)$, DNA-DNA homology data $(13,20,29)$, rRNA-DNA homology data $(8,9,11,52-54), 16 \mathrm{~S}$ rRNA sequence data $(24,40,56-59)$, and 5 S rRNA sequence data (7, $12,41)$, and it is clear that the five groups of Palleroni et al. differ at least at the genus level.

Many plant-associated or plant-pathogenic strains of Pseudomonas species $(36,42,46)$, particularly strains of Pseudomonas cepacia $(1,4,51)$, Pseudomonas gladioli $(1,4,16$, 51), Pseudomonas glumae (51), and Pseudomonas avenae (17, 35,51 , have been isolated from various kinds of plants and have been identified as bacteria that exhibit growth-inhibiting activity against plant-pathogenic microorganisms, such as Clavibacter michiganensis subsp. michiganensis, Agrobacterium tumefaciens, Fusarium oxysporum f. sp. cepae, and other organisms. $P$. cepacia strains appear to be widely distributed in nature and have been isolated from rotten onions, soils, and clinical specimens (e.g., urinary tract infection specimens) (28). Furthermore, $P$. avenae ("Pseudomonas alboprecipitans" [38], Pseudomonas rubrilineans [35]) and Pseudomonas cattleyae ("Bacterium cattleyae") (2) were isolated as plant-associated or plant-pathogenic bacteria.

\footnotetext{
* Corresponding author. Mailing address: Biochemicals Division, Mitsubishi Gas Chemical Co., Seavans-N Building, Shibaura, Minatoku, Tokyo 105, Japan. Phone: (03) 5440-3218. Fax: (03) 5440-3229.

$\dagger$ Present address: Department of Agricultural Chemistry, Tokyo University of Agriculture, Sakuragaoka 1-1-1, Setagaya-ku, Tokyo 156, Japan.
}

P. cepacia, P. gladioli ("Pseudomonas marginata"), Pseudomonas caryophylli, Pseudomonas solanacearum (5, 15), Pseudomonas pickettii $(23,33)$, Pseudomonas mallei, and Pseudomonas pseudomallei were included in rRNA group II of the genus Pseudomonas by Palleroni et al. (32) and are identified as section II bacteria in Bergey's Manual of Systematic Bacteriology (28). $P$. glumae was included in rRNA group II on the basis of the rRNA-DNA hybridization data of De Vos et al. (9). Pseudomonas plantarii was isolated as the causal agent of rice seedling blight by Azegami et al. and was shown to be related to $P$. gladioli (3). Recently, the rRNA sequences of several Pseudomonas species were studied $(7,12,24,40,41,56-59)$, and $P$. cepacia, a typical species of rRNA group II, was assigned to the $\beta$-purple bacteria (40). This phylogenetic characterization supported the differentiation of Pseudomonas rRNA group II bacteria (28) (P. solanacearum rRNA complex in rRNA superfamily III [11]) from Pseudomonas rRNA group I bacteria (28) (Pseudomonas fluorescens rRNA branch in rRNA superfamily II [11]) at least at the genus level. In 1992, Yabuuchi et al. (60) proposed that seven species belonging to Pseudomonas rRNA group II ( $P$. cepacia, $P$. mallei, $P$. pseudomallei, $P$. caryophylli, $P$. gladioli, $P$. picketti, and $P$. solanacearum) should be transferred to a new genus, Burkholderia, on the basis of polyphasic taxonomy data. $P$. avenae and $P$. cattleyae were included in section V of the genus Pseudomonas in Bergey's Manual of Systematic Bacteriology (28). The results of rRNA-DNA hybridization experiments performed with $P$. avenae and $P$. cattleyae $(9,11,55)$ demonstrated that these bacteria belong in the Pseudomonas acidovorans rRNA complex in rRNA superfamily III $(11,28)$. In 1992 , Willems et al. (54) proposed the transfer of $P$. avenae and $P$. cattleyae to the new genus Acidovorax (53) as Acidovorax avenae subsp. avenae and Acidovorax avenae subsp. cattleyae, respectively.

In this paper, we describe a detailed recharacterization of plant-associated bacteria, clarify the differentiation of these 
TABLE 1. Bacterial strains studied

\begin{tabular}{|c|c|c|}
\hline Strain $^{a}$ & Other designations $^{a}$ & Reference(s) \\
\hline $\begin{array}{l}\text { Burkholderia cepacia ATCC } 25416^{\mathrm{T}} \\
\text { Burkholderia cepacia ALO } 8281^{b}\end{array}$ & ICP $25^{\mathrm{T}}, \mathrm{JCM} 5964^{\mathrm{T}}$, NCTC $10743^{\mathrm{T}}$ & $\begin{array}{l}4,28,31,60 \\
60\end{array}$ \\
\hline Burkholderia gladioli ATCC $10248^{\mathrm{T}}$ & IFO $13700^{\mathrm{T}}$, NCPPB $1891^{\mathrm{T}}$, PDDCC $2804^{\mathrm{T}}$ & $4,16,28,60,62$ \\
\hline Burkholderia gladioli ATCC 19302 & NCPPB 947, ICPB PA7, PDDCC 2804 & $4,16,28,60,62$ \\
\hline Pseudomonas plantarii $\mathrm{JCM} 5492^{\mathrm{T}}$ & NIAES $1723^{\mathrm{T}}$ & 3 \\
\hline Burkholderia caryophylli ATCC $25418^{\mathrm{T}}$ & ICPB PC113 ${ }^{\mathrm{T}}$, NCPPB $2151^{\mathrm{T}}$, PDDCC $512^{\mathrm{T}}$ & $4,28,60$ \\
\hline Pseudomonas glumae NIAES $1169^{\mathrm{T}}$ & ATCC $33617^{\mathrm{T}}$, NCPPB $2981^{\mathrm{T}}$ & 28,46 \\
\hline Burkholderia pickettii JCM $5969^{\mathrm{T}}$ & ATCC $27511^{\mathrm{T}}$, ICPB $3981^{\mathrm{T}}$ & $23,28,34,36,60$ \\
\hline Burkholderia solanacearum ATCC $11696^{\mathrm{T}}$ & NCPPB $325^{\mathrm{T}}$, PDDCC $5721^{\mathrm{T}}$ & $5,15,23,28,30,60$ \\
\hline Acidovorax avenae subsp. avenae ATCC $19860^{\mathrm{T}}$ & NCPPB $1011^{\mathrm{T}}$ & $28,35,38,54$ \\
\hline Acidovorax avenae subsp. cattleyae ATCC $33619^{\mathrm{T}}$ & NCPPB $961^{\mathrm{T}}$ & $2,28,54$ \\
\hline Pseudomonas aeruginosa JCM $5962^{\mathrm{T}}$ & ATCC $10145^{\mathrm{T}}$, NCIB $8295^{\mathrm{T}}$, NCTC $10332^{\mathrm{T}}$ & 28 \\
\hline Pseudomonas fluorescens NCIB $9046^{\mathrm{T}}$ & $\begin{array}{l}\text { ATCC } 13525^{\mathrm{T}} \text {, IAM } 12022^{\mathrm{T}}, \text { ICPB } 3200^{\mathrm{T}} \text {, IFO } 14160^{\mathrm{T}}, \text { JCM } \\
5963^{\mathrm{T}}, \text { NCPPB } 1964^{\mathrm{T}}\end{array}$ & 28 \\
\hline Isolate VA- $1316^{\mathrm{T}}$ & & This study \\
\hline Isolate D-2251 & & This study \\
\hline Isolate CY-0619 & & This study \\
\hline Isolate CY-0627 & & This study \\
\hline Isolate VU-0563 & & This study \\
\hline
\end{tabular}

a Abbreviations: ATCC, American Type Culture Collection, Rockville, Md.; ICPB, International Collection of Phytopathogenic Bacteria, University of California, Davis; IFO, Institute for Fermentation, Osaka, Japan; JCM, Japan Collection of Microorganisms, The Institute of Physical and Chemical Research, Wako-shi, Saitama, Japan; NCIB, National Collection of Industrial Bacteria, Torry Research Station, Aberdeen, United Kingdom; NCPPB, National Collection of Plant Pathogenic Bacteria, Harpenden, United Kingdom; NCTC, National Collection of Type Cultures, London, United Kingdom; NIAES, National Institute of Agro-Environmental Science, Ibaragi, Japan; PDDCC, Plant Disease Division Culture Collection, Auckland, New Zealand.

${ }^{b}$ This bacterium was supplied by M. Goto, Faculty of Agriculture, Shizuoka University, Shizuoka, Japan.

bacteria from authentic members of the genus Pseudomonas sensu stricto, and propose the transfer of $P$. plantarii and $P$. glumae to the genus Burkholderia and the establishment of a new species, Burkholderia vandii.

\section{MATERIALS AND METHODS}

Bacterial strains. The strains which we studied are shown in Table 1. Names which do not appear on the Approved Lists of Bacterial Names (39) are enclosed in quotation marks. Strains VA-1316 ${ }^{\mathrm{T}}$ ( $\mathrm{T}=$ type strain), D-2251, CY-0619, CY-0627, and VU-0563 were isolated by us as antibiotic-producing bacteria that are active against $C$. michiganensis subsp. michiganensis. Strain VA- $1316^{\mathrm{T}}$ was isolated from roots of Vanda species, strain D-2251 was isolated from roots of Dendrobium species, strains CY-0619 and CY-0627 were isolated from roots of Cymbidium species, and strain VU-0563 was isolated from roots of Vuylstekeara species. These bacteria were maintained on PYG agar (47).

Morphological, biochemical, and physiological characterization. PYG agar was used as the preculture and basal medium for bacterial strains. Unless indicated otherwise, the strains were cultivated at $30^{\circ} \mathrm{C}$. Morphological, biochemical, and physiological characteristics were investigated by using methods described previously (47). Production of indole was determined with Kovács reagent in $1 \%$ tryptone broth (catalog no. 0123; Difco Laboratories, Detroit, Mich.). Production of hydrogen sulfide was tested on triple sugar iron agar for 2 weeks. The requirement for vitamins was tested in liquid basal medium B (49), and methanol was replaced with $1 \%(\mathrm{wt} / \mathrm{vol})$ D-glucose or $1 \%(\mathrm{wt} / \mathrm{vol}) \mathrm{D}$-galactose. Utilization of carbon compounds was determined in liquid basal medium $\mathrm{B}$, and methanol was replaced with other carbon compounds as reported previously (47). In addition, we used the same method to determine the utilization of L-rhamnose, adonitol, glycine, leucine, L-isoleucine, L-valine, L-threonine, ornithine, DL-norleucine, DL-citrulline, sarcosine, $n$-butanol, isobutanol, $n$-propanol, propylene glycol, 2,3-butanediol, monoethanol- amine, $n$-amylamine, tryptamine, benzylamine, acetoamide, suberic acid, levulinic acid, mesaconic acid, L-tartaric acid, D-tartaric acid, itaconic acid, pimelic acid, citraconic acid, caprylic acid, nicotinic acid, sebacic acid, benzoylformic acid, L-mandelic acid, 4-hydroxy-2-quinoline carboxylic acid, $n$-capric acid, glutaric acid, DL-2-aminobutyric acid, $n$-caproic acid, $n$-heptanoic acid, benzoic acid, $p$-aminobenzoic acid, $m$-hydroxybenzoic acid, adipic acid, salicylic acid, $n$-nonanoic acid, azelaic acid, and glycolic acid, except that each of these compounds was tested at a concentration of $0.15 \%$ (wt/vol).

Cellular fatty acid and hydroxy fatty acid compositions. Cells cultivated in PYG medium ( $\mathrm{pH} \mathrm{7.0)}$ at $30^{\circ} \mathrm{C}$ for 1 day with shaking were used to determine cellular fatty acid and hydroxy fatty acid compositions by methods described previously $(48,50)$.

Quinone systems and quinone homologs. Cells cultivated in PYG medium ( $\mathrm{pH} 7.0$ ) at $30^{\circ} \mathrm{C}$ for 2 days with shaking were used to determine the quinone systems as described previously $(48,49)$.

DNA base composition. DNA was extracted by the method of Saito and Miura (37), and the guanine-plus-cytosine $(\mathrm{G}+\mathrm{C})$ content was determined by reversed-phase high-performance liquid chromatography (45).

DNA-DNA hybridization. DNA-DNA hybridization was carried out at $68^{\circ} \mathrm{C}$ by using the method of Kaneko et al. (21). DNAs of Burkholderia cepacia ATCC $25416^{\mathrm{T}}$, Burkholderia gladioli ATCC $10248^{\mathrm{T}}, P$. glumae NIAES $1169^{\mathrm{T}}$, and new isolate VA- $1316^{\mathrm{T}}$ were labelled with $\left(1^{\prime}, 2^{\prime}, 5^{\prime}-{ }^{3} \mathrm{H}\right) \mathrm{dCTP}$ by the nick translation method, using an Amersham kit (catalog no. TRK 700; Amersham International plc, Amersham, United Kingdom). DNA-DNA hybridization experiments were performed with the Pseudomonas rRNA group II bacteria, our new isolates, Acidovorax avenae subsp. avenae ATCC $19860^{\mathrm{T}}$, Acidovorax avenae subsp. cattleyae ATCC $33619^{\mathrm{T}}$, and Pseudomonas aeruginosa JCM $5962^{\mathrm{T}}$.

Resistance to antibiotic compounds. Resistance to antibiotic compounds by Pseudomonas strains was tested by using Showa 
TABLE 2. Differentiating physiological characteristics of plant-associated bacteria

\begin{tabular}{|c|c|c|c|c|c|c|c|c|c|}
\hline \multirow{2}{*}{ Strain } & \multirow{2}{*}{ 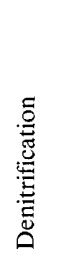 } & \multirow{2}{*}{ 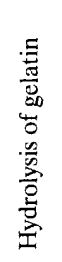 } & \multirow{2}{*}{ 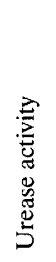 } & \multirow{2}{*}{ 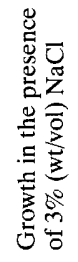 } & \multicolumn{2}{|c|}{$\begin{array}{l}\text { Utilization of } \\
\text { the following } \\
\text { nitrogen } \\
\text { sources: }\end{array}$} & \multicolumn{3}{|c|}{ Growth at: } \\
\hline & & & & & 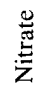 & $\stackrel{\Xi}{5}$ & $\mathrm{pH} 4$ & $\mathrm{pH} 8$ & $\mathrm{pH} 9$ \\
\hline B. cepacia ATCC $25416^{\mathrm{T}}$ & $-a$ & - & + & $\mathrm{w}$ & $w$ & + & + & + & - \\
\hline B. cepacia ALQ 8281 & - & - & + & + & w & + & + & + & - \\
\hline B. gladioli ATCC $10248^{\mathrm{T}}$ & - & w & + & - & + & + & + & + & - \\
\hline B. gladioli ATCC 19302 & - & + & + & - & w & w & + & + & - \\
\hline Isolate D-2251 & - & + & + & w & + & + & + & + & - \\
\hline Isolate CY-0619 & w & w & + & - & w & $\mathrm{w}$ & + & + & - \\
\hline Isolate CY-0627 & - & + & - & w & $\mathrm{w}$ & w & + & + & - \\
\hline Isolate VU-0563 & - & w & - & - & + & + & + & + & - \\
\hline Isolate VA-1316 ${ }^{\mathrm{T}}$ & + & + & + & - & - & + & + & $\mathrm{w}$ & - \\
\hline P. plantarii JCM $5492^{\mathrm{T}}$ & + & + & + & - & w & w & + & - & - \\
\hline B. caryophylli ATCC $25418^{\mathrm{T}}$ & + & - & - & - & - & + & - & - & - \\
\hline P. glumae NIAES $1169^{\mathrm{T}}$ & + & + & + & + & w & w & - & w & - \\
\hline B. solanacearum ATCC $11696^{\mathrm{T}}$ & - & - & + & - & - & $\mathrm{w}$ & - & $\mathrm{w}$ & - \\
\hline B. pickettii JCM $5969^{\mathrm{T}}$ & + & - & + & - & - & - & - & + & - \\
\hline Acidovorax avenae subsp. avenae ATCC $19860^{\mathrm{T}}$ & + & - & + & - & - & - & - & + & + \\
\hline Acidovorax avenae subsp. cattleyae ATCC $33619^{\mathrm{T}}$ & - & - & + & - & - & - & - & + & $\mathrm{w}$ \\
\hline
\end{tabular}

${ }^{a}+$, positive; - , negative; $\mathbf{w}$, weakly positive.

disks (diameter, 9 mm; Showa Yakuhin Kakou, Tokyo, Japan). Each disk contained $30 \mu \mathrm{g}$ of cefotiam hydrochloride, $30 \mu \mathrm{g}$ of cefmetazole (sodium salt), $30 \mu \mathrm{g}$ of cefotaxime (sodium salt), $30 \mu \mathrm{g}$ of cefmetanoxin sulfate, $30 \mu \mathrm{g}$ of lotamoxysefu (sodium salt), $30 \mu \mathrm{g}$ of ceftizoxime (sodium salt), $30 \mu \mathrm{g}$ of aminobenzylpenicillin, $30 \mu \mathrm{g}$ of gentamicin sulfate, $30 \mu \mathrm{g}$ of amikacin sulfate, or $200 \mu \mathrm{g}$ of minocycline hydroxychloride.

Production of antibiotic substances. To detect the production of antibiotic substances, C. michiganensis subsp. michiganensis and $F$. oxysporum f. sp. lycopersici $\mathrm{J}-3$, which are distantly related to each other, were used as indicator organisms. These indicator organisms are known to be distributed widely and to cause serious plant diseases. The inhibitory activities of the test strains against the growth of $C$. michiganensis were studied by the following method. Each strain tested was streaked onto nutrient agar overlaid with $C$. michiganensis, the preparation was incubated at $30^{\circ} \mathrm{C}$, and the size of the growth inhibition zone formed on the agar plate was measured. To test the inhibitory activities of the test strains against the growth of $F$. oxysporum, each strain tested and $F$. oxysporum were streaked separately on PDA agar (Nissui, Tokyo, Japan), the preparation was incubated for 7 days at $25^{\circ} \mathrm{C}$, and inhibition of $F$. oxysporum growth by the test strain was measured.

TABLE 3. Oxidation of sugars by plant-associated bacteria

\begin{tabular}{|c|c|c|c|c|c|c|c|c|c|c|c|c|c|c|c|}
\hline \multirow[b]{2}{*}{ Strain } & \multicolumn{15}{|c|}{ Oxidation of: } \\
\hline & 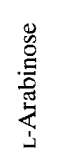 & 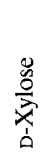 & 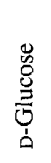 & 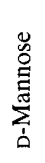 & 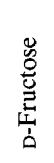 & 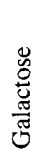 & 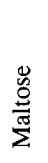 & 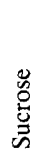 & 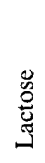 & 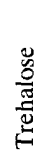 & 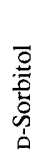 & 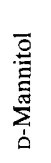 & 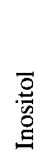 & $\begin{array}{l}\overline{0} \\
\text { 岕 } \\
\text { 品 }\end{array}$ & 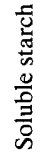 \\
\hline B. cepacia ATCC $25416^{\mathrm{T}}$ & $-{ }^{a}$ & - & + & - & - & + & - & + & - & + & - & - & - & - & - \\
\hline B. cepacia ALQ 8281 & - & - & + & - & - & + & - & + & - & - & - & - & - & - & - \\
\hline B. gladioli ATCC $10248^{\mathrm{T}}$ & + & + & + & + & + & + & - & - & - & + & - & - & - & - & - \\
\hline B. gladioli ATCC 19302 & + & + & + & + & + & + & - & - & - & + & - & - & - & - & - \\
\hline Isolate D-2251 & + & + & + & + & + & + & - & - & - & - & - & - & - & - & - \\
\hline Isolate CY-0619 & + & + & + & + & + & + & - & - & - & - & - & - & - & $w$ & - \\
\hline Isolate CY-0627 & + & + & + & + & + & + & - & - & - & + & - & - & - & - & - \\
\hline Isolate VU-0563 & + & + & + & + & + & + & - & - & - & + & - & - & - & - & - \\
\hline Isolate VA-1316 ${ }^{\mathrm{T}}$ & - & - & - & - & - & - & - & - & - & - & - & - & w & w & - \\
\hline P. plantarii $\mathrm{JCM} 5492^{\mathrm{T}}$ & + & + & + & + & + & + & - & - & - & - & - & - & - & w & - \\
\hline B. caryophylli ATCC $25418^{\mathrm{T}}$ & - & - & - & - & - & - & - & - & - & - & - & - & - & w & - \\
\hline$P$. glumae NIAES $1169^{\mathrm{T}}$ & - & - & - & - & - & - & - & - & - & - & - & - & - & $\mathrm{w}$ & - \\
\hline B. solanacearum ATCC $11696^{\mathrm{T}}$ & - & - & - & - & - & - & - & - & - & - & - & - & - & $\mathrm{w}$ & - \\
\hline B. pickettii JCM $5969^{\mathrm{T}}$ & - & - & + & $w$ & - & w & + & - & $\mathrm{w}$ & - & - & - & - & - & - \\
\hline Acidovorax avenae subsp. avenae ATCC $19860^{\mathrm{T}}$ & - & - & - & - & - & + & - & - & - & - & - & - & - & w & - \\
\hline Acidovorax avenae subsp. cattleyae ATCC $33619^{\mathrm{T}}$ & - & $\mathrm{w}$ & - & - & - & + & - & - & - & - & - & - & - & w & - \\
\hline
\end{tabular}

${ }^{a}+$, positive; - , negative; w, weakly positive. 
TABLE 4. Utilization of carbon compounds by plant-associated bacteria

\begin{tabular}{|c|c|c|c|c|c|c|c|c|c|c|c|c|c|c|c|c|c|}
\hline & 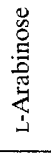 & $\begin{array}{c}\mathscr{y} \\
\stackrel{0}{\grave{\lambda}} \\
\dot{a} \\
\dot{b}\end{array}$ & $\begin{array}{l}0 \\
0 \\
0 \\
0 \\
0 \\
\dot{0} \\
\dot{0}\end{array}$ & 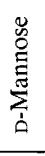 & 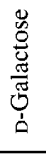 & 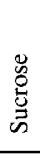 & 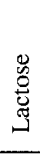 & 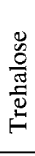 & 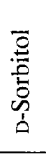 & 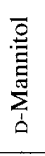 & $\begin{array}{l}\overrightarrow{\underline{a}} \\
\stackrel{0}{00} \\
\stackrel{g}{g}\end{array}$ & 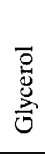 & 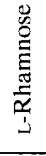 & 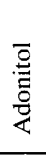 & $\begin{array}{l}\frac{2}{0} \\
\frac{2}{0} \\
0\end{array}$ & 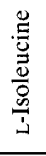 & 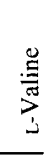 \\
\hline B. cepacia ATCC $25416^{\mathrm{T}}$ & $+^{a}$ & + & + & + & + & + & $\mathrm{w}$ & + & + & + & + & + & - & + & w & + & + \\
\hline B. серасіа ALQ 8281 & + & + & + & + & + & + & - & + & + & + & + & + & - & + & - & + & + \\
\hline B. gladioli ATCC $10248^{\mathrm{T}}$ & + & + & + & + & + & - & $\mathrm{w}$ & + & + & + & + & + & - & + & - & + & + \\
\hline B. gladioli ATCC 19302 & + & + & + & + & + & - & $\mathrm{w}$ & + & + & + & + & + & - & + & + & + & + \\
\hline Isolate D-2251 & + & + & + & + & + & - & $\mathrm{w}$ & + & + & + & + & + & - & + & w & + & + \\
\hline Isolate CY-0619 & + & + & + & + & + & - & - & + & + & + & + & + & - & + & w & + & + \\
\hline Isolate CY-0627 & + & + & + & + & + & - & - & + & + & + & + & + & - & + & - & + & + \\
\hline Isolate VU-0563 & + & + & + & + & + & - & $\mathrm{w}$ & + & + & + & + & + & - & + & - & + & + \\
\hline Isolate VA- $1316^{\mathrm{T}}$ & + & + & + & + & + & - & $\mathrm{w}$ & + & + & + & + & + & - & - & - & + & + \\
\hline P. plantarii JCM $5492^{\mathrm{T}}$ & + & + & + & + & + & $\mathrm{w}$ & - & - & + & + & + & + & w & - & - & - & $\mathrm{w}$ \\
\hline B. caryophylli ATCC $25418^{\mathrm{T}}$ & + & + & + & + & + & + & - & + & + & + & + & + & - & - & - & $\mathrm{w}$ & + \\
\hline P. glumae NIAES $1169^{\mathrm{T}}$ & + & + & + & + & + & - & - & + & + & + & + & $\mathrm{w}$ & - & + & - & $\mathrm{w}$ & $\mathrm{w}$ \\
\hline B. solanacearum ATCC $11696^{\mathrm{T}}$ & - & - & + & - & - & + & - & - & - & - & + & + & - & - & - & + & + \\
\hline B. pickettii JCM $5969^{\mathrm{T}}$ & + & + & + & - & + & - & - & - & - & - & - & + & - & - & w & + & + \\
\hline Acidovorax avenae subsp. avenae ATCC $19860^{T}$ & + & - & - & - & + & - & - & - & + & + & - & + & - & - & - & - & - \\
\hline Acidovorax avenae subsp. cattleyae ATCC $33619^{\mathrm{T}}$ & + & - & - & - & + & - & - & - & + & + & - & + & - & - & - & + & + \\
\hline
\end{tabular}

${ }^{a}+$, positive;,- negative; w, weakly positive.

Tolerance to fusaric acid. Most strains tested inhibited the growth of $F$. oxysporum f. sp. lycopersici J-3. Meanwhile, $F$. oxysporum produced fusaric acid, which inhibits the growth of microorganisms (22). Therefore, the tolerance of the test strains to fusaric acid was investigated. Fusaric acid was added to PYG broth at a concentration of 0.01 to $1.0 \%$ (wt/vol).

\section{RESULTS}

Phenotypic characteristics of the test strains. All strains were gram negative, non-spore-forming, rod-shaped organisms whose cells were 0.5 to $1.0 \mu \mathrm{m}$ wide by 1.5 to $3.0 \mu \mathrm{m}$ long and had rounded ends. The cells occurred singly, rarely in pairs, and were motile by means of one or several polar flagella. Colonies were white to light yellow. Abundant growth was observed in nutrient broth, PYG broth, and peptone water. No water-soluble fluorescent pigment was produced on King B medium. Only $P$. glumae produced a fluorescent pigment on potato agar. Granules of poly- $\beta$-hydroxybutyrate accumulated in the cells. Nitrate was reduced to nitrite by all strains. The methyl red test and the Voges-Proskauer reaction were negative. Production of indole in 1\% tryptone broth (Difco) and production of hydrogen sulfide in triple sugar iron medium were not observed. Hydrolysis of starch was not observed. Ammonia was produced in peptone water. Litmus milk was not changed. Catalase and oxidase were produced. All strains produced acid from sugars oxidatively. However, the kinds of sugars that produced acid were not identical to the kinds of sugars that produced acid in previous studies $(15,28,60)$ for reasons which are not clear. The acid formation patterns of $B$. gladioli, $P$. plantarii, and four new isolates (but not isolate VA-1316 ${ }^{\mathrm{T}}$ ) resembled each other. All strains utilized D-fruc- tose, L-leucine, succinic acid, acetic acid, sebacic acid, and adipic acid, but did not utilize maltose, soluble starch, methanol, monomethylamine, dimethylaminme, trimethylamine, $N, N$-dimethylformamide, methane, hydrogen, and formic acid. Growth factors were not required as essential supplements. All strains utilized ammonia and peptone as sole nitrogen sources. All strains grew at $\mathrm{pH} 5.0$ to 7.5 , but did not grow at $\mathrm{pH}$ values below 3.0 or above 9.5 . All strains grew at 30 and $37^{\circ} \mathrm{C}$ but not at $42^{\circ} \mathrm{C}$. Biochemical and physiological characteristics that differentiate strains are shown in Tables 2 to 4 . The rRNA group II organisms B. cepacia, B. gladioli, P. plantarii, Burkholderia caryophylli, P. glumae, Burkholderia solanacearum, and Burkholderia pickettii and Acidovorax avenae strains were distinguished from each other on the basis of biochemical and physiological characteristics (Tables 2 to 4). New isolates D-2251, CY-0619, CY-0627, and CY-0563 were identical to $B$. gladioli on the basis of phenotypic characteristics, but new isolate VA- $1316^{\mathrm{T}}$ differed from the other strains.

Cellular fatty acid compositions. The cellular fatty acids of all of the strains tested consisted of large amounts of $n-\mathrm{C}_{16: 0}$, $n-\mathrm{C}_{16: 1}$, and $n-\mathrm{C}_{18: 1}$ acids (Table 5).

Hydroxy fatty acid compositions. All of the Pseudomonas rRNA group II bacteria except $B$. solanacearum and $B$. pickettii and the new isolates contained large amounts of 3-hydroxy $\mathrm{C}_{14: 0}$, 3-hydroxy $\mathrm{C}_{16: 0}$, and 2-hydroxy $\mathrm{C}_{16: 0}$ acids as their hydroxy fatty acid components. $B$. solanacearum contained large amounts of 3-hydroxy $\mathrm{C}_{14: 0}$ and 2-hydroxy $\mathrm{C}_{16: 0}$ acids. $B$. pickettii contained large amounts of 3-hydroxy $\mathrm{C}_{14: 0}$, 2-hydroxy $\mathrm{C}_{14: 0}$, and 2-hydroxy $\mathrm{C}_{16: 0}$ acids. In contrast, Acidovorax avenae strains contained a large amount of 3-hydroxy $\mathrm{C}_{10: 0}$ acid, and the Pseudomonas rRNA group I bacteria ( $P$. aeruginosa and $P$. 
TABLE 4-Continued

\begin{tabular}{|c|c|c|c|c|c|c|c|c|c|c|c|c|c|c|c|c|c|c|c|c|c|c|c|}
\hline 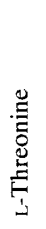 & 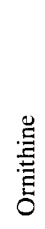 & 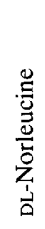 & 总 & 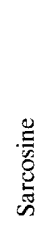 & $\begin{array}{l}\overline{0} \\
\text { 䭴 } \\
\text { 吉 }\end{array}$ & 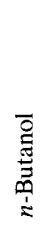 & 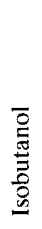 & $\begin{array}{l}\overline{0} \\
\bar{\Xi} \\
\bar{\Xi} \\
\stackrel{0}{\Xi} \\
\vdots\end{array}$ & 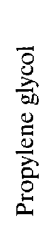 & 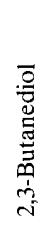 & 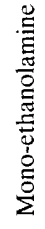 & 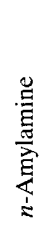 & 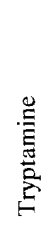 & 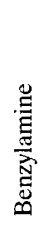 & 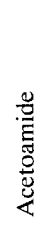 & 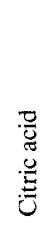 & 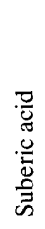 & 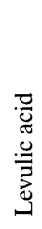 & 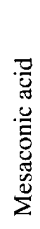 & 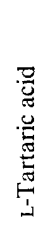 & 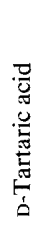 & 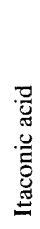 & 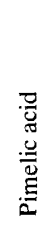 \\
\hline+ & + & + & w & w & + & + & w & + & $\mathrm{w}$ & + & + & + & + & + & + & w & + & + & - & w & $w$ & - & + \\
\hline+ & + & $w$ & w & w & $\mathrm{w}$ & + & w & + & w & + & + & + & + & + & + & w & + & + & - & $\mathrm{w}$ & $\mathbf{w}$ & - & + \\
\hline+ & + & - & + & + & w & w & - & - & + & - & + & - & + & w & + & $\mathrm{w}$ & $\mathbf{w}$ & $w$ & + & $\mathrm{w}$ & + & - & w \\
\hline+ & + & $w$ & $\therefore-$ & - & w & w & w & $\mathrm{w}$ & + & - & + & - & - & - & - & $\mathrm{w}$ & $w$ & w & w & w & $\mathrm{w}$ & w & \\
\hline+ & + & + & - & + & w & + & - & - & + & - & w & - & - & + & - & $w$ & $\mathrm{w}$ & - & - & $\mathrm{w}$ & $\mathrm{w}$ & + & w \\
\hline+ & + & $w$ & - & + & $\mathrm{w}$ & + & - & - & + & - & w & - & - & + & - & w & w & + & + & $\mathrm{w}$ & w & - & + \\
\hline+ & - & w & - & + & + & + & - & - & + & - & w & - & - & w & - & $\mathrm{w}$ & w & w & + & w & $\mathrm{w}$ & - & + \\
\hline+ & - & + & - & + & w & + & - & - & $w$ & - & + & - & - & - & - & w & w & + & + & w & $\mathrm{w}$ & - & w \\
\hline+ & w & - & - & + & + & - & - & + & w & - & - & - & - & - & - & - & - & - & + & - & $\mathrm{w}$ & - & - \\
\hline- & - & $w$ & - & w & + & w & - & $\mathrm{w}$ & - & - & $\mathbf{w}$ & - & - & - & $w$ & $w$ & - & - & $w$ & $\mathrm{w}$ & - & - & - \\
\hline- & - & - & - & - & - & w & - & w & w & + & - & + & - & + & - & $w$ & w & - & - & - & - & - & - \\
\hline- & - & w & - & w & + & + & - & + & - & - & + & - & + & - & - & w & - & + & - & - & - & - & w \\
\hline+ & - & - & - & - & - & w & - & + & - & - & - & - & - & - & - & w & + & + & - & - & - & - & $w$ \\
\hline+ & - & + & - & - & - & + & - & - & - & - & - & - & - & - & - & w & + & + & - & w & - & - & + \\
\hline- & - & w & - & - & + & + & - & + & + & + & - & - & - & - & - & - & + & w & - & - & & & \\
\hline- & - & - & - & - & + & + & - & + & + & + & $\mathrm{W}$ & - & - & - & w & - & + & + & - & - & - & - & + \\
\hline
\end{tabular}

fluorescens) contained 3-hydroxy $\mathrm{C}_{10: 0}$, 3-hydroxy $\mathrm{C}_{12: 0}$, and 2-hydroxy $\mathrm{C}_{12: 0}$ acids (Table 6).

Quinone systems and quinone homologs. Pseudomonas rRNA group II and III bacteria and all of the new isolates contained a large amount of ubiquinone Q-8. In contrast, the Pseudomonas rRNA group I organisms $P$. aeruginosa and $P$. fluorescens contained large amounts of ubiquinone Q-9 (Table 6 ). These results are consistent with the data of Yamada et al. (61) and Oyaizu and Komagata (27).

DNA base compositions. The DNA base compositions of all of the Pseudomonas rRNA group II bacteria, our new isolates, and Acidovorax avenae strains ranged from 64 to $70 \mathrm{~mol} \%$ $\mathrm{G}+\mathrm{C}$ (Table 6). The $\mathrm{G}+\mathrm{C}$ contents of $P$. aeruginosa and $P$. fluorescens were 65.9 and $60.2 \mathrm{~mol} \%$, respectively.

DNA-DNA hybridization. The levels of DNA-DNA similarity among Pseudomonas rRNA group II bacteria, our new isolates, Acidovorax avenae strains, and $P$. aeruginosa are shown in Table 7. The Pseudomonas rRNA group II bacteria and our new isolates were clearly distinguished from $A c$ idovorax avenae strains and $P$. aeruginosa. Our new isolates were clearly divided into two groups. Four of our new isolates (D-2251, CY-0619, CY-0627, and VU-0563) exhibited high similarity values ( 68 to $82 \%$ ) with $B$. gladioli, but new isolate VA-1316 ${ }^{\mathrm{T}}$ exhibited low similarity values (17 to $57 \%$ ) with Pseudomonas rRNA group II bacteria. B. solanacearum and $B$. pickettii exhibited less than $20 \%$ similarity with other Pseudomonas rRNA group II bacteria and our new isolates.

Resistance to antibiotic compounds. All of the strains except $P$. aeruginosa and $P$. fluorescens strains and our new isolates were susceptible to cefotaxime (sodium salt), cefmetanoxin sulfate, ceftizoxime (sodium salt), gentamicin sulfate, amikacin sulfate, and minocycline hydroxychloride. B. gladioli, $P$. plantarii, $P$. glumae, B. solanacearum, Acidovorax avenae subsp. cattleyae, and all of our new isolates were susceptible to all of the antibiotic compounds tested. B. cepacia strains were resistant to cefotiam hydrochloride and aminobenzylpenicillin, $B$. caryophylli was resistant to cefotiam hydrochloride, cefmetazole (sodium salt), lotamoxysefu (sodium salt), B. pickettii was resistant to cefotiam hydrochloride, and Acidovorax avenae subsp. avenae was resistant to aminobenzylpenicillin.

Antibiotic activity against plant-pathogenic microorganisms. Some saprophytic Pseudomonas strains are known to produce antibiotics, pyrrolnitrin (produced by $B$. cepacia) and/or pyoluteorin $(18,25,26,51)$. Most Pseudomonas rRNA group II strains and all of our new isolates inhibited the growth of the plant-pathogenic microorganisms $C$. michiganensis subsp. michiganensis and $F$. oxysporum f. sp. lycopersici J-3 (Table 8). Figure 1 shows the growth inhibition zone formed by new isolate VA-1316 ${ }^{\mathrm{T}}$ on a lawn of $C$. michiganensis subsp. michiganensis. However, Acidovorax avenae strains did not inhibit the growth of plant-pathogenic microorganisms.

Tolerance to fusaric acid. All Pseudomonas rRNA group II strains and our new isolates grew in medium containing $0.01 \%$ (wt/vol) fusaricacid, but Acidovorax avenae strains did not grow in this medium (Table 8). All of the Pseudomonas rRNA group II bacteria except $B$. solanacearum and $B$. pickettii and all of our new isolates grew in medium containing $0.05 \%$ (wt/vol) fusaric acid.

\section{DISCUSSION}

Pseudomonas rRNA group II strains, B. cepacia, B. gladioli, $P$. plantarii, B. caryophylli, $P$. glumae, $B$. solanacearum, $B$. pickettii, and our new isolates are plant-associated bacteria that have the same morphological characteristics. However, the results of our study of utilization of carbon compounds (D- 
TABLE 4-Continued

\begin{tabular}{|c|c|c|c|c|c|c|c|c|c|c|c|c|c|c|c|c|c|c|}
\hline \multirow[b]{2}{*}{ Strain } & \multicolumn{18}{|c|}{ Utilization of: } \\
\hline & 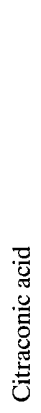 & 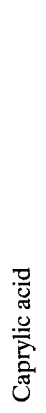 & 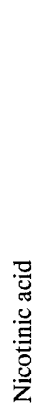 & 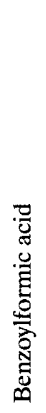 & 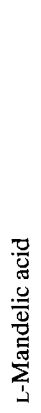 & 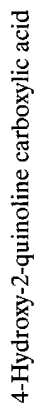 & 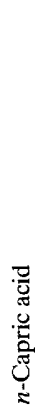 & 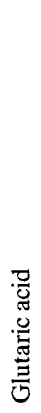 & 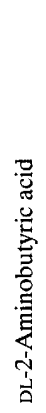 & 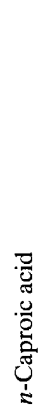 & 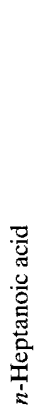 & 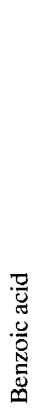 & 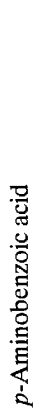 & 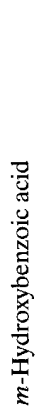 & 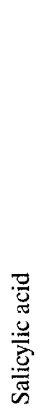 & 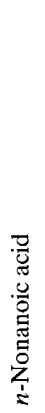 & 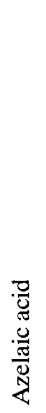 & 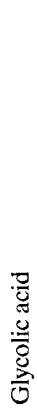 \\
\hline B. cepacia ATCC $25416^{\mathrm{T}}$ & + & + & - & + & + & + & + & + & + & + & + & + & + & $\mathrm{w}$ & + & + & + & + \\
\hline B. cepacia ALQ 8281 & + & + & - & + & + & + & + & + & w & + & + & + & + & w & + & + & + & + \\
\hline B. gladioli ATCC $10248^{\mathrm{T}}$ & - & + & + & - & - & + & + & - & - & + & + & + & + & + & - & + & + & - \\
\hline B. gladioli ATCC 19302 & + & + & + & - & - & + & + & - & w & + & + & + & + & - & - & + & + & - \\
\hline Isolate D-2251 & - & + & + & - & - & + & + & - & + & + & + & + & + & - & - & + & + & - \\
\hline Isolate CY-0619 & + & + & + & - & - & + & + & - & + & + & + & + & + & - & - & + & + & - \\
\hline Isolate CY-0627 & + & + & + & - & - & + & + & - & + & + & - & + & + & - & - & + & + & - \\
\hline Isolate VU-0563 & + & + & + & - & - & + & + & - & + & + & + & + & + & - & - & + & + & - \\
\hline Isolate VA-1316 ${ }^{\mathrm{T}}$ & + & + & + & - & - & + & + & - & - & - & + & - & - & - & - & + & - & - \\
\hline P. plantarii JCM $5492^{\mathrm{T}}$ & w & w & + & - & - & w & w & - & - & w & + & - & - & - & - & + & w & - \\
\hline B. caryophylli ATCC $25418^{\mathrm{T}}$ & - & - & - & - & - & - & - & w & - & - & - & w & - & - & - & - & w & + \\
\hline P. glumae NIAES $1169^{\mathrm{T}}$ & - & + & $\mathbf{w}$ & - & - & - & w & - & - & w & + & - & w & - & + & - & + & - \\
\hline B. solanacearum ATCC $11696^{\mathrm{T}}$ & - & - & - & - & - & - & - & - & - & + & + & - & - & - & - & - & + & - \\
\hline B. pickettii JCM $5969^{\mathrm{T}}$ & - & - & - & + & + & - & - & - & - & - & - & - & - & - & - & - & - & - \\
\hline Acidovorax avenae subsp. avenae ATCC $19860^{\mathrm{T}}$ & - & - & - & - & - & - & - & + & $\mathrm{w}$ & $\mathrm{w}$ & - & - & - & - & - & - & + & - \\
\hline Acidovorax avenae subsp. cattleyae ATCC $33619^{\mathrm{T}}$ & w & - & - & - & - & - & - & + & - & + & - & - & - & - & - & - & + & - \\
\hline
\end{tabular}

xylose, L-rhamnose, and L-valine for B. cepacia; sucrose and glycine for $B$. gladioli; L-rhamnose, trehalose, L-valine, and sebacic acid for B. caryophylli; L-leucine for B. solanacearum; and L-leucine for $B$. pickettii) were different from those described in Bergey's Manual of Systematic Bacteriology (28).
Furthermore, the data for acid formation from sugars obtained in this study were different from the data obtained by Yabuuchi et al. (60). The reasons for these unexpected results are not apparent. These bacteria have the same major cellular fatty acid and ubiquinone system components. Galbraith and

TABLE 5. Cellular fatty acid compositions of plant-associated bacteria

\begin{tabular}{|c|c|c|c|c|c|c|c|c|c|c|c|c|c|c|c|c|c|c|}
\hline \multirow{3}{*}{ Strain } & \multicolumn{18}{|c|}{$\%$ of total acids } \\
\hline & \multicolumn{9}{|c|}{ Straight-chain acids } & \multicolumn{2}{|c|}{$\begin{array}{l}\text { Cyclopro- } \\
\text { pane acids }\end{array}$} & \multicolumn{4}{|c|}{ 3-Hydroxy acids } & \multicolumn{3}{|c|}{ 2-Hydroxy acids } \\
\hline & $\mathrm{C}_{12: 0}$ & $\mathrm{C}_{14: 0}$ & $\mathrm{C}_{15: 0}$ & $\mathrm{C}_{16: 0}$ & $\mathrm{C}_{16: 1}$ & $\mathrm{C}_{17: 0}$ & $\mathrm{C}_{18: 0}$ & $\mathrm{C}_{18: 1}$ & $\mathrm{C}_{19: 0}$ & $\mathrm{C}_{17: 0}$ & $C_{19: 0}$ & $\begin{array}{r}3-\mathrm{OH} \\
\mathrm{C}_{10: 0}\end{array}$ & $\begin{array}{l}3-\mathrm{OH} \\
\mathrm{C}_{12: 0} \\
\end{array}$ & $\begin{array}{l}3-\mathrm{OH} \\
\mathrm{C}_{14: 0}\end{array}$ & $\begin{array}{c}3-\mathrm{OH} \\
\mathrm{C}_{16: 0}\end{array}$ & $\begin{array}{r}2-\mathrm{OH} \\
\mathrm{C}_{12: 0}\end{array}$ & $\begin{array}{r}2-\mathrm{OH} \\
\mathrm{C}_{14: 0}\end{array}$ & $\begin{array}{r}2-\mathrm{OH} \\
\mathrm{C}_{16: 0} \\
\end{array}$ \\
\hline B. cepacia ATCC $25416^{\mathrm{T}}$ & & 3.6 & 0.1 & 25.4 & 22.9 & 0.1 & 0.5 & 41.6 & & 3.1 & 1.0 & & & 0.4 & 0.3 & & & 1.0 \\
\hline B. cepacia ALQ 8281 & 0.1 & 3.5 & 0.1 & 23.4 & 25.2 & 0.1 & 1.0 & 41.0 & & 2.3 & 0.6 & & & 1.2 & 0.5 & & & 1.0 \\
\hline B. gladioli ATCC $10248^{\mathrm{T}}$ & & 3.6 & & 26.6 & 22.4 & 0.1 & 1.2 & 39.1 & & 3.0 & 0.9 & & & 1.6 & 0.5 & & & 1.0 \\
\hline B. gladioli ATCC 19302 & & 3.8 & & 28.0 & 24.9 & & 0.7 & 37.8 & & 2.9 & 0.7 & & & 0.4 & 0.1 & & & 0.7 \\
\hline Isolate D-2251 & & 3.9 & 0.1 & 27.8 & 22.4 & 0.2 & 1.2 & 33.4 & 1.6 & 2.3 & 0.6 & & & 3.8 & 1.3 & & & 1.4 \\
\hline Isolate CY-0619 & & 3.7 & 0.2 & 26.4 & 22.6 & 0.3 & 1.1 & 39.3 & & 1.7 & 0.4 & & & 2.7 & 0.8 & & & 0.8 \\
\hline Isolate CY-0627 & & 3.8 & 0.2 & 27.7 & 22.9 & 0.3 & 1.2 & 36.3 & & 2.4 & 0.5 & & & 2.8 & 0.9 & & & 1.0 \\
\hline Isolate VU-0563 & & 4.0 & 0.1 & 26.7 & 21.7 & 0.2 & 1.3 & 38.6 & & 2.1 & 0.6 & & & 2.5 & 0.7 & & & 1.5 \\
\hline Isolate VA-1316 & & 4.1 & & 27.8 & 19.7 & 0.1 & 1.3 & 34.7 & 0.6 & 4.7 & 0.7 & & & 3.4 & 2.1 & & & 0.8 \\
\hline P. plantarï JCM $5492^{\mathrm{T}}$ & & 4.2 & 0.1 & 25.9 & 21.2 & 0.6 & 1.0 & 29.0 & 0.8 & 3.8 & 3.6 & & & 2.5 & 0.1 & & & 2.0 \\
\hline B. caryophylli ATCC $25418^{\mathrm{T}}$ & & 3.1 & 0.4 & 22.0 & 15.0 & 0.6 & 1.2 & 48.1 & & 1.7 & 0.5 & & & 2.5 & 0.9 & & & 4.0 \\
\hline P. glumae NIAES $1169^{\mathrm{T}}$ & & 4.6 & 0.2 & 28.1 & 22.6 & 0.4 & 0.9 & 33.4 & 0.3 & 2.3 & 1.3 & & & 4.5 & 0.9 & & & 0.6 \\
\hline B. solanacearum ATCC $11696^{\mathrm{T}}$ & & 4.2 & 1.0 & 25.5 & 28.4 & 1.9 & 0.9 & 30.4 & 0.3 & 5.8 & 0.2 & & & 0.4 & & & & 1.0 \\
\hline B. pickettii JCM $5969^{\mathrm{T}}$ & & 3.3 & 0.6 & 27.2 & 31.0 & 2.0 & 0.9 & 33.0 & & 1.3 & & & & 0.3 & & & 0.2 & 0.2 \\
\hline $\begin{array}{l}\text { Acidovorax avenae subsp. avenae } \\
\text { ATCC } 19860^{\mathrm{T}}\end{array}$ & 2.4 & 2.1 & 0.5 & 33.7 & 45.4 & 0.2 & 0.3 & 13.8 & & 0.7 & & 0.9 & & & & & & \\
\hline $\begin{array}{l}\text { Acidovorax avenae subsp. cattleyae } \\
\text { ATCC } 33619^{\mathrm{T}}\end{array}$ & 2.0 & 1.3 & 0.9 & 33.4 & 42.4 & 0.6 & 0.3 & 14.4 & & 4.0 & & 0.7 & & & & & & \\
\hline P. aeruginosa JCM 5962 & & 1.1 & & 23.2 & 19.6 & & 0.3 & 46.8 & & 0.5 & 0.3 & 2.3 & 0.6 & & & 5.3 & & \\
\hline P. fluorescens NCIB $9046^{\mathrm{T}}$ & & 1.6 & & 30.8 & 35.2 & & 1.5 & 22.0 & & 0.1 & 0.2 & 3.0 & 1.1 & & & 4.5 & & \\
\hline
\end{tabular}


TABLE 6. DNA base compositions, ubiquinone homologs, and hydroxy fatty acid compositions of plant-associated bacteria

\begin{tabular}{|c|c|c|c|c|c|c|c|c|c|c|c|c|}
\hline \multirow{2}{*}{ Strain } & \multirow{2}{*}{$\begin{array}{c}\mathrm{G}+\mathrm{C} \\
\text { content } \\
\text { (mol\%) }\end{array}$} & \multicolumn{4}{|c|}{$\%$ of total ubiquinones } & \multicolumn{4}{|c|}{$\begin{array}{c}\% \text { of total 3-hydroxy } \\
\text { fatty acids }\end{array}$} & \multicolumn{3}{|c|}{$\begin{array}{l}\% \text { of total 2-hydroxy } \\
\text { fatty acids }\end{array}$} \\
\hline & & Q-7 & Q-8 & Q-9 & Q-10 & $\begin{array}{l}3-\mathrm{OH} \\
\mathrm{C}_{10: 0}\end{array}$ & $\begin{array}{l}3-\mathrm{OH} \\
\mathrm{C}_{12: 0}\end{array}$ & $\begin{array}{r}3-\mathrm{OH} \\
\mathrm{C}_{14: 0}\end{array}$ & $\begin{array}{l}3-\mathrm{OH} \\
\mathrm{C}_{16: 0}\end{array}$ & $\begin{array}{l}2-\mathrm{OH} \\
\mathrm{C}_{12: 0}\end{array}$ & $\begin{array}{c}2-\mathrm{OH} \\
\mathrm{C}_{14: 0}\end{array}$ & $\begin{array}{l}2-\mathrm{OH} \\
\mathrm{C}_{16: 0}\end{array}$ \\
\hline B. cepacia ATCC $25416^{\mathrm{T}}$ & 67.1 & 0.9 & 98.2 & 0.9 & & & & 72.8 & 27.3 & & & 100 \\
\hline B. cepacia ALQ 8281 & & 2.7 & 96.6 & 0.7 & & & & 72.7 & 27.3 & & & 100 \\
\hline B. gladioli ATCC $10248^{\mathrm{T}}$ & 67.9 & 0.9 & 98.2 & 0.9 & & & & 77.1 & 22.9 & & & 100 \\
\hline B. gladioli ATCC 19302 & 68.3 & 0.9 & 98.2 & 0.9 & & & & 73.4 & 26.6 & & & 100 \\
\hline Isolate D-2251 & 69.4 & 0.6 & 98.9 & 0.5 & & & & 74.8 & 25.2 & & & 100 \\
\hline Isolate CY-0619 & 68.8 & 0.5 & 98.8 & 0.7 & & & & 77.5 & 22.5 & & & 100 \\
\hline Isolate CY-0627 & 68.5 & 0.3 & 98.9 & 0.8 & & & & 76.4 & 23.6 & & & 100 \\
\hline Isolate VU-0563 & 69.5 & 0.4 & 98.6 & 1.0 & & & & 77.5 & 22.5 & & & 100 \\
\hline Isolate VA- $1316^{\mathrm{T}}$ & 68.5 & 1.6 & 97.9 & 0.7 & & & & 61.6 & 38.4 & & & 100 \\
\hline P. plantarii $\mathrm{JCM} 5492^{\mathrm{T}}$ & 68.7 & 3.9 & 95.7 & 0.4 & & & & 64.9 & 35.1 & & & 100 \\
\hline B. caryophylli ATCC $25418^{\mathrm{T}}$ & 65.2 & 2.3 & 97.6 & 0.1 & & & & 74.1 & 25.9 & & & 100 \\
\hline P. glumae NIAES $1169^{\mathrm{T}}$ & 68.2 & 2.0 & 97.4 & 0.6 & & & 1.3 & 81.9 & 16.8 & & & 100 \\
\hline B. solanacearum ATCC $11696^{\mathrm{T}}$ & 66.6 & 3.3 & 96.4 & 0.3 & & & & 100 & & & & 100 \\
\hline B. pickettii JCM $5969^{\mathrm{T}}$ & 64.0 & 1.5 & 98.1 & 0.4 & & & & 97.3 & 2.7 & & 47.2 & 52.8 \\
\hline Acidovorax avenae subsp. avenae ATCC $19860^{\mathrm{T}}$ & 69.5 & 1.2 & 96.0 & 2.8 & & 100 & & & & & & \\
\hline Acidovorax avenae subsp. cattleyae ATCC $33619^{\mathrm{T}}$ & 69.0 & 1.1 & 94.1 & 4.8 & & 100 & & & & & & \\
\hline P. aeruginosa JCM $5962^{\mathrm{T}}$ & 65.9 & 0.3 & 6.2 & 92.8 & 0.7 & 69.9 & 30.1 & & & 100 & & \\
\hline P. fluorescens NCIB $9046^{\mathrm{T}}$ & 60.2 & & 4.0 & 92.9 & 3.1 & 66.3 & 33.4 & & & 100 & & \\
\hline
\end{tabular}

Wilkinson (14) reported that the nonhydroxy fatty acids of $B$. caryophylli and $B$. gladioli consisted of large amounts of $n-C_{16: 0}$, $n-\mathrm{C}_{18: 1}$, and cyclopropane $\mathrm{C}_{17: 0}$ acids and that $B$. pickettii contained large amounts of $n-C_{16: 0}, n-C_{16: 1}, n-C_{18: 1}$, and cyclopropane $C_{17: 0}$ acids. The differences in our results may have been due to the culture age of the cells used, because a decrease in the amount of $n-C_{16: 1}$ acid and an increase in the amount of cyclopropane $C_{17: 0}$ acid with age have been reported previously (48). On the other hand, Stead (43) and Janse (19) described the cellular fatty acid compositions of Pseudomonas species, and their results for nonhydroxy fatty acid composition and 3-hydroxy fatty acid composition were identical to our results. However, their results for 2-hydroxy fatty acid composition were different from our results. The failure to detect 2-OH $\mathrm{C}_{16: 1}$ and 2-OH C 18 :1 acids in this study may have been due to a difference in the detection limits of these 2-hydroxy fatty acids. These bacteria (B. cepacia, $B$. gladioli, B. plantarii, B. caryophylli, $P$. glumae, and our new isolates) were identical to each other in physiological characteristics, ubiquinone systems, cellular fatty acid compositions, and hydroxy fatty acid compositions. On the basis of phenotypic and chemotaxonomic characteristics, $P$. plantarii and $P$. glumae should be included in the genus Burkholderia. New isolates D-2251, CY-0619, CY-0627, and VU-0563 were identified as $B$. gladioli strains on the basis of physiological characteristics (Tables 2 to 4 ) and DNA-DNA similarity values (Table 7). On the other hand, the DNA-DNA similarity values obtained for new isolate VA-1316 ${ }^{\mathrm{T}}, P$. glumae NIAES $1169^{\mathrm{T}}$, and $P$. plantarii JCM $5492^{\mathrm{T}}$ were approximately 51 to $57 \%$, and these values are at the borderline for the separating species. However, these three strains could be clearly distinguished from each other on the basis of carbon compound utilization patterns. Therefore, we believe that new isolate VA-1316 should be separated from previously described Burkholderia species at the species level, and we propose a new species for this strain. Recently, Li et al. (24) studied the 16S rRNA

TABLE 7. Levels of DNA-DNA homology among plant-associated bacteria

\begin{tabular}{|c|c|c|c|c|}
\hline \multirow[b]{2}{*}{ Strain } & \multicolumn{4}{|c|}{ \% DNA-DNA homology with: } \\
\hline & $\begin{array}{c}\text { Strain ATCC } \\
25416^{\mathrm{T}}\end{array}$ & $\begin{array}{c}\text { Strain ATCC } \\
10248^{\mathrm{T}}\end{array}$ & $\begin{array}{c}\text { Strain } \\
\text { VA-1316 }\end{array}$ & $\begin{array}{c}\text { Strain NIAES } \\
1169^{\mathrm{T}}\end{array}$ \\
\hline B. cepacia ATCC $25416^{\mathrm{T}}$ & 100 & 23 & 24 & 23 \\
\hline B. gladioli ATCC $10248^{\mathrm{T}}$ & 30 & 100 & 45 & 41 \\
\hline B. gladioli ATCC 19302 & 26 & 68 & 40 & 37 \\
\hline Isolate D-2251 & 31 & 81 & 46 & 44 \\
\hline Isolate CY-0619 & 26 & 68 & 40 & 38 \\
\hline Isolate CY-0627 & 29 & 80 & 45 & 41 \\
\hline Isolate VU-0563 & 29 & 82 & 44 & 37 \\
\hline Isolate VA-1316 ${ }^{\mathrm{T}}$ & 30 & 42 & 100 & 55 \\
\hline P. glumae NIAES $1169^{\mathrm{T}}$ & 27 & 37 & 51 & 100 \\
\hline P. plantarii JCM $5492^{\mathrm{T}}$ & 25 & 33 & 57 & 48 \\
\hline B. caryophylli ATCC $25418^{\mathrm{T}}$ & 21 & 18 & 23 & 19 \\
\hline B. solanacearum ATCC $11696^{\mathrm{T}}$ & 14 & 17 & 17 & 16 \\
\hline B. pickettii JCM $5969^{\mathrm{T}}$ & 15 & 15 & 17 & 14 \\
\hline Acidovorax avenae subsp. avenae ATCC $19860^{\mathrm{T}}$ & 8 & 7 & 8 & 6 \\
\hline Acidovorax avenae subsp. cattleyae ATCC $33619^{\mathrm{T}}$ & 7 & 8 & 8 & 8 \\
\hline P. aeruginosa JCM $5962^{\mathrm{T}}$ & 6 & 9 & 6 & 9 \\
\hline
\end{tabular}


TABLE 8. Production of an antibiotic substance and tolerance to fusaric acid

\begin{tabular}{|c|c|c|c|c|c|c|}
\hline \multirow{2}{*}{ Strain } & \multicolumn{2}{|c|}{$\begin{array}{l}\text { Activity against the following } \\
\text { indicators: }\end{array}$} & \multicolumn{4}{|c|}{$\begin{array}{l}\text { Tolerance to a fusaric } \\
\text { acid concn of: }\end{array}$} \\
\hline & C. michiganensis ${ }^{a}$ & F. oxysporum ${ }^{b}$ & $0.01 \%(\mathrm{wt} / \mathrm{vol})$ & $0.05 \%(\mathrm{wt} / \mathrm{vol})$ & $0.1 \%(\mathrm{wt} / \mathrm{vol})$ & $0.5 \%(\mathrm{wt} / \mathrm{vol})$ \\
\hline B. cepacia ATCC $25416^{\mathbf{T}}$ & ++ & + & + & + & + & - \\
\hline B. cepacia ALQ 8281 & ++ & + & + & + & + & - \\
\hline B. gladioli ATCC $10248^{\mathrm{T}}$ & + & + & + & + & + & + \\
\hline B. gladioli ATCC 19302 & - & + & + & + & + & + \\
\hline Isolate D-2251 & + & + & + & + & + & + \\
\hline Isolate CY-0619 & + & + & + & + & + & + \\
\hline Isolate CY -0627 & + & + & + & + & + & + \\
\hline Isolate VU-0563 & ++ & + & + & + & + & - \\
\hline Isolate VA-1316 & $+t$ & + & + & + & + & - \\
\hline P. plantarii JCM $5492^{\mathrm{T}}$ & - & + & + & + & + & + \\
\hline B. caryophylli ATCC $25418^{\mathrm{T}}$ & - & - & + & + & - & - \\
\hline$P$. glumae NIAES $1169^{\mathrm{T}}$ & + & + & + & + & + & - \\
\hline B. solanacearum ATCC $11696^{\mathrm{T}}$ & - & - & + & - & - & - \\
\hline B. pickettii JCM $5969^{\mathrm{T}}$ & - & - & + & - & - & - \\
\hline $\begin{array}{l}\text { Acidovorax avenae subsp. avenae } \\
\text { ATCC } 19860^{\mathrm{T}}\end{array}$ & - & - & - & - & - & - \\
\hline $\begin{array}{l}\text { Acidovorax avenae subsp. cattleyae } \\
\text { ATCC } 33619^{\mathrm{T}}\end{array}$ & - & - & - & - & - & - \\
\hline
\end{tabular}

${ }^{a}-$, activity not detected; + , width of inhibition zone less than $5 \mathrm{~mm} ;++$, width inhibition zone more than $5 \mathrm{~mm}$

$b$ _, activity not detected; + , growth inhibition observed.

sequences of the rRNA group II pseudomonads and found evidence which suggested a dichotomy in this group. One subgroup contained Pseudomonas andropogonis, B. caryophylli, and $B$. gladioli, and the second subgroup contained $B$. solanacerum and B. pickettii. Furthermore, Stead (43) reported that $P$. solanacerum could be distinguished from other Pseudomonas rRNA group II bacteria on the basis of hydroxy fatty acid composition. These results were supported by the DNADNA similarity and hydroxy fatty acid composition data obtained in this study. Therefore, we believe that the genus

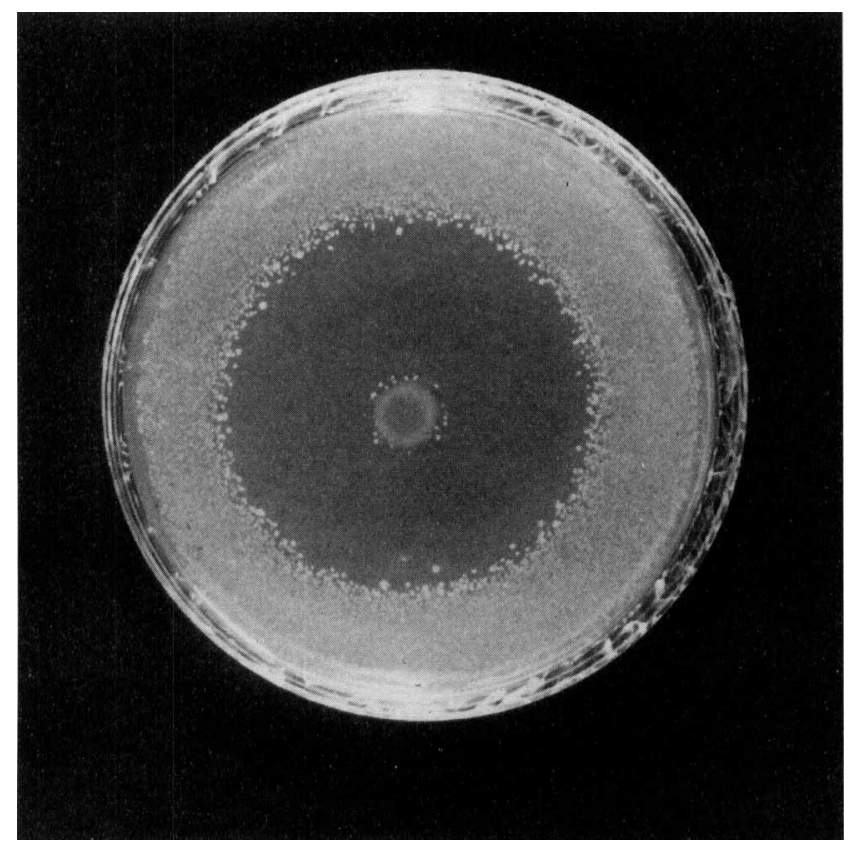

FIG. 1. Growth inhibition zone formed by isolate VA-1316 ${ }^{\mathrm{T}}$ on a lawn of $C$. michiganensis subsp. michiganensis.
Burkholderia should be characterized more precisely in the future.

Acidovorax avenae subsp. avenae and Acidovorax avenae subsp. cattleyae had the same chemotaxonomic characteristics and could be distinguished clearly from the genus Pseudomonas sensu stricto ( $P$. fluorescens rRNA branch [11]), Burkholderia species, Comamonas species, and Hydrogenophaga species on the basis of phenotypic characteristics $(10,17,28,35,38$, 44), ubiquinone systems, DNA base compositions, DNA-DNA homology data, and rRNA-DNA hybridization data $(9,11,52$, 53) (Table 9). Minimal characteristics for differentiating the genera Burkholderia, Acidovorax, Comamonas, Hydrogenophaga, and Pseudomonas are shown in Table 9. Characteristics which differentiate among Burkholderia species are shown in Tables 3 to 7 and are described in Bergey's Manual of Systematic Bacteriology (28).

Transfer of Pseudomonas plantarii and Pseudomonas glumae to the genus Burkholderia as Burkholderia species. Pseudomonas plantarii was transferred previously to the genus Burkholderia as Burkholderia plantarii (Azegami, Nishiyama, Watanabe, Kadota, Ohuchi, and Fukazawa 1987) comb. nov. This species is described in this study and in reference 3 , and the type strain is strain JCM 5492 (= NIAES 1723). Pseudomonas glumae has also been transferred to the genus Burkholderia as Burkholderia glumae (Uematsu, Yoshimura, Nishiyama, Ibaraki, and Fujii 1976) comb. nov. This species is described in this study and in references 28 and 46, and the type strain is NIAES 1169 $(=$ ATCC $33617=$ NCPPB 2981).

Description of Burkholderia vandii Urakami, Ito-Yoshida, Araki, Kijima, Suzuki, and Komagata sp. nov. Burkholderia vandii (vand' i. i. M.L. adj. vandii, coming from Hindi Vanda.) Non-spore-forming, gram-negative, rod-shaped organisms. The cells are 0.5 to 1.0 by 1.5 to $3.0 \mu \mathrm{m}$ and have rounded ends. The cells occur singly, rarely in pairs, and are motile by means of one or several polar flagella. Granules of poly- $\beta$-hydroxybutyrate accumulate in the cells. Colonies are white to light yellow. No water-soluble fluorescent pigment is produced. Abundant growth occurs in nutrient broth, PYG broth, and peptone water. Nitrate is reduced to nitrite. The methyl red 
TABLE 9. Characteristics that differentiate the genera of plant-associated bacteria from Pseudomonas strains

\begin{tabular}{|c|c|c|c|c|c|c|c|c|}
\hline Genus & Typical species & $\begin{array}{l}\text { Quinone } \\
\text { system }\end{array}$ & $\begin{array}{l}\text { Major cellular } \\
\text { fatty acids }\end{array}$ & $\begin{array}{l}\text { Major 3-hydroxy } \\
\text { acid(s) }\end{array}$ & $\begin{array}{c}\text { Major } \\
\text { 2-hydroxy } \\
\text { acid }\end{array}$ & $\begin{array}{l}\mathrm{G}+\mathrm{C} \\
\text { content } \\
(\mathrm{mol} \%)\end{array}$ & $\begin{array}{l}\text { rRNA } \\
\text { group }\end{array}$ & $\begin{array}{c}16 \mathrm{~S} \\
\text { rRNA } \\
\text { sequence }\end{array}$ \\
\hline Burkholderia & B. cepacia & Q-8 & $\mathrm{C}_{16: 0}, \mathrm{C}_{16: 1}, \mathrm{C}_{1}$ & $3-\mathrm{OH} \mathrm{C} \mathrm{C}_{14: 0}\left(3-\mathrm{OH} \mathrm{C} \mathrm{C}_{16: 0}\right)$ & $2-\mathrm{OH} \mathrm{C} \mathrm{C}_{16: 0}$ & $67-68$ & III-2 & $\beta-2$ \\
\hline Acidovorax & A. avenae & Q-8 & $C_{16: 0}, C_{16: 1}\left(C_{18: 1}\right)$ & $3-\mathrm{OH} \mathrm{C}$ & $\mathrm{ND}^{c}$ & $70-71$ & III-1 & \\
\hline Comamonas & C. acidovorans ${ }^{d}$ & Q-8 & $\mathrm{C}_{16: 0}, \mathrm{C}_{16: 1}, \mathrm{C}_{18: 1}$ & $3-\mathrm{OH} \mathrm{C} 10: 0$ & $2-\mathrm{OH} \mathrm{C} \mathrm{C}_{16: 0}$ & $66-67$ & III-1 & $\beta-1$ \\
\hline Hydrogenophaga & H. flava ${ }^{e}$ & Q-8 & $\mathrm{C}_{16: 0}, \mathrm{C}_{16: 1}, \mathrm{C}_{18: 1}$ & $3-\mathrm{OH} \mathrm{C} 10: 0$ & ND & $66-67$ & III-1 & $\beta-1$ \\
\hline Pseudomonas & P. aeruginosa & Q-9 & $C_{16: 0}, C_{16: 1}, C_{18: 1}$ & $3-\mathrm{OH} \mathrm{C} \mathrm{C}_{10: 0}\left(3-\mathrm{OH} \mathrm{C} \mathrm{C}_{12: 0}\right)$ & $2-\mathrm{OH} \mathrm{C} \mathrm{C}_{12: 0}$ & $65-66$ & II-1 & $\gamma-3$ \\
\hline
\end{tabular}

${ }^{a}$ Data from studies of De Vos et al. $(9,11)$ and Willems et al. $(52-55)$. Group II-1, $P$. fluorescens rRNA branch in rRNA superfamily II; group III-1, P. acidovorans rRNA complex in rRNA superfamily III; group III-2, $P$. solanacearum rRNA complex in rRNA superfamily III.

${ }^{b}$ Data from references 56 through 59.

ND, not detected.

${ }^{d}$ Data from reference 44

${ }^{e}$ Data from references 27 and 52.

test and the Voges-Proskauer reaction are negative. Indole and hydrogen sulfide are not produced. Starch is not hydrolyzed. Ammonia is produced. Litmus milk is not changed. Positive for dentrification and hydrolysis of gelatin. Acids are weakly produced from inositol and glycerol oxidatively, but are not produced from L-arabinose, D-xylose, D-glucose, D-mannose, D-fructose, D-galactose, maltose, sucrose, lactose, trehalose, D-sorbitol, D-mannitol, and soluble starch. Acid is not produced from sugars fermentatively. L-Arabinose, D-xylose, Dglucose, D-fructose, D-mannose, D-galactose, lactose, trehalose, D-sorbitol, D-mannitol, inositol, glycerol, L-leucine, L-isoleucine, L-threonine, ornithine, sarcosine, ethanol, $n$-propanol, propylene glycol, succinic acid, acetic acid, sebacic acid, adipic acid, mesaconic acid, D-tartaric acid, citraconic acid, caprylic acid, nicotinic acid, 4-hydroxy-2-quinoline carboxylic acid, $n$ capric acid, $n$-heptanoic acid, and $n$-nonanoic acid are utilized, but sucrose, L-rhamnose, maltose, soluble starch, adonitol, glycine, DL-norleucine, DL-citrulline, methanol, n-butanol, isobutanol, 2,3-butanediol, monoethanolamine, $n$-amylamine, tryptamine, benzylamine, acetoamide, formic acid, citric acid, suberic acid, levulinic acid, L-tartaric acid, itaconic acid, pimelic acid, benzoylformic acid, L-mandelic acid, glutaric acid, DL-2-aminobutyric acid, $n$-caproic acid, benzoic acid, $p$-aminobenzoic acid, $m$-hydroxybenzoic acid, salicylic acid, azelaic acid, glycolic acid, monomethylamine, dimethylamine, trimethylamine, $N, N$-dimethylformamide, methane, and hydrogen are not utilized. Growth factors are not required. Ammonia, urea, and peptone are utilized as nitrogen sources, but nitrate is not utilized. Oxidase, catalase, and urease are produced. Aerobic. Metabolism is strictly respiratory and not fermentative. Good growth occurs between pH 5.0 and 7.5, and weak growth occurs at $\mathrm{pH} 4.0$ and 8.0. Growth does not occur at $\mathrm{pH}$ values above 9.0 and below 3.0. Good growth occurs at 30 and $37^{\circ} \mathrm{C}$, but does not occur at $42^{\circ} \mathrm{C}$. Growth does not occur in the presence of $3 \%$ sodium chloride. Susceptible to cefotaxime, cefmetanoxim, ceftizoxime, gentamicin, amikacin, minocycline, cefotiam, cefmetazole, lotamoxysefu, and aminobenzylpenicillin. Growth occurs in the presence of $0.1 \%$ (wt/vol) fusaric acid. Growing cells inhibit the growth of the phytopathogenic microorganisms $C$. michiganensis and $F$. oxysporum. The cellular fatty acids include large amounts of straight-chain saturated $n-\mathrm{C}_{16: 0}$ acid and unsaturated $n-\mathrm{C}_{16: 1}$ and $\mathrm{C}_{18: 1}$ acids. The hydroxy acids include large amounts of $3-\mathrm{OH} \mathrm{C}_{14: 0}$ and 2-OH $\mathrm{C}_{16: 0}$ acids. The ubiquinone system is Q-8.

The type strain is strain VA-1316. Strain VA- $1316^{\mathrm{T}}$ has all of the characteristics described above for the species. The DNA base composition is $68.5 \mathrm{~mol} \% \mathrm{G}+\mathrm{C}$. This strain was isolated from Vanda species by Urakami et al. as a antibiotic-producing bacterium active against $C$. michiganensis subsp. michiganensis.
The type strain has been deposited in the Japan Collection of Microorganisms, Institute of Physical and Chemical Research, under accession number JCM 7957.

\section{ACKNOWLEDGMENTS}

We thank M. Goto (Faculty of Agriculture, Shizuoka University) for supplying B. cepacia ALQ 8281.

\section{REFERENCES}

1. Arie, T., S. Namba, S. Yamashita, Y. Doi, and T. Kijima. 1987. Biological control of Fusarium wilt of bottle gourd by mixcropping with welsh onion or Chinese chive inoculated with Pseudomonas gladioli. Ann. Phytopathol. Soc. Jpn. 53:531-539.

2. Ark, P. A., and H. E. Thomas. 1946. Bacterial leaf spot and bud rot of orchids caused by Phytomonas cattleyae. Phytopathology 36: 695-698.

3. Azegami, K., K. Nishiyama, Y. Watanabe, I. Kadota, A. Ohuchi, and C. Fukazawa. 1987. Pseudomonas plantarii sp. nov., the causal agent of rice seedling blight. Int. J. Syst. Bacteriol. 37:144-152.

4. Ballard, R. W., N. J. Palleroni, M. Doudoroff, R. Y. Stanier, and M. Mandel. 1970. Taxonomy of the aerobic pseudomonads: Pseudomonas cepacia, $P$. marginata, $P$. allicola, and $P$. caryophylli. J. Gen. Microbiol. 60:199-214.

5. Buddenhagen, I. W., and A. Kelman. 1964. Biological and physiological aspects of bacterial wilt caused by Pseudomonas solanacearum. Annu. Rev. Phytopathol. 2:203-230.

6. Byng, G. S., R. J. Whitaker, R. L. Gherna, and R. A. Jensen. 1980. Variable enzymological patterning in tyrosine biosynthesis as a means of determining natural relatedness among the Pseudomonadaceae. J. Bacteriol. 144:247-257.

7. Dams, E., A. Vandenberghe, and R. D. Wachter. 1983. Sequences of the 5S rRNAs of Azotobacter vinelandii, Pseudomonas aeruginosa and Pseudomonas fluorescens with some notes on 5S RNA secondary structure. Nucleic Acids Res. 11:1245-1252.

8. De Vos, P., and J. De Ley. 1983. Intra- and intergeneric similarities of Pseudomonas and Xanthomonas ribosomal ribonucleic acid cistrons. Int. J. Syst. Bacteriol. 33:487-509.

9. De Vos, P., M. Goor, M. Gillis, and J. De Ley. 1985. Ribosomal ribonucleic acid cistron similarities of phytopathogenic Pseudomonas species. Int. J. Syst. Bacteriol. 35:169-184.

10. De Vos, P., K. Kersters, E. Falsen, B. Pot, M. Gillis, P. Segers, and J. De Ley. 1985. Comamonas Davis and Park 1962 gen. nov., nom. rev. emend., and Comamonas terrigena Hugh 1962 sp. nov., nom. rev. Int. J. Syst. Bacteriol. 35:443-453.

11. De Vos, P., A. Van Landschoot, P. Segers, R. Tytgat, M. Gillis, M. Bauwens, R. Rossau, M. Goor, B. Pot, K. Kersters, P. Lizzaraga, and J. De Ley. 1989. Genotypic relationships and taxonomic localization of unclassified Pseudomonas and Pseudomonas-like strains by deoxyribonucleic acid-ribosomal ribonucleic acid hybridizations. Int. J. Syst. Bacteriol. 39:35-49.

12. Erdmann, V. A., and J. Wolters. 1986. Collection of published 5S, $5.8 \mathrm{~S}$ and $4.5 \mathrm{~S}$ ribosomal RNA sequences. Nucleic Acids Res. 14:r1-r59. 
13. Ezaki, T., Y. Hashimoto, and E. Yabuuchi. 1989. Fluorometric deoxyribonucleic acid-deoxyribonucleic acid hybridization in microdilution wells as an alternative to membrane filter hybridization in which radioisotopes are used to determine genetic relatedness among bacterial strains. Int. J. Syst. Bacteriol. 39:224-229.

14. Galbraith, L., and S. G. Wilkinson. 1991. Polar lipids and fatty acids of Pseudomonas caryophylli, Pseudomonas gladioli and Pseudomonas picketti. J. Gen. Microbiol. 137:197-202.

15. Hayward, A. C. 1964. Characteristics of Pseudomonas solanacearum. J. Appl. Bacteriol. 27:265-277.

16. Hildebrand, D. C., N. J. Palleroni, and M. Doudoroff. 1973. Synonymy of Pseudomonas gladioli Severini 1913 and Pseudomonas marginata (McCulloch 1921) Stapp 1928. Int. J. Syst. Bacteriol. 23:433-437.

17. Hu, F.-P., J. M. Young, and C. M. Triggs. 1991. Numerical analysis and determinative tests for nonfluorescent plant-pathogenic Pseudomonas spp. and genomic analysis and reclassification of species related to Pseudomonas avenae Manns 1909. Int. J. Syst. Bacteriol. 41:516-525.

18. Imanaka, H., M. Kousaka, G. Tamura, and K. Arima. 1965. Studies on pyrrolnitrin, a new antibiotic. II. J. Antibiot. Ser. A 18:205-206.

19. Janse, J. D. 1991. Infra- and intraspecific classification of Pseudomonas solanacearum strains, using whole cell fatty acid analysis. Syst. Appl. Microbiol. 14:335-345.

20. Johnson, J. L., and N. J. Palleroni. 1989. Deoxyribonucleic acid similarities among Pseudomonas species. Int. J. Syst. Bacteriol. 39:230-235

21. Kaneko, T., R. Nozaki, and K. Aizawa. 1978. Deoxyribonucleic acid relatedness between Bacillus anthracis, Bacillus cereus, and Bacillus thuringiensis. Microbiol. Immunol. 22:639-641.

22. Kern, H. 1972. Phytotoxins produced by fusaria, p. 35-48. In Phytotoxins in plant diseases. Academic Press, New York.

23. King, A., B. Holmes, I. Phillips, and S. P. Lapage. 1979. A taxonomic study of clinical isolates of Pseudomonas pickettii, ' $P$. thomasii' and 'group IVd' bacteria. J. Gen. Microbiol. 114:137147.

24. Li, X., M. Dorsch, D. T. Del, L. I. Sly, E. Stackebrandt, and A. C. Hayward. 1993. Phylogenetic studies of the rRNA group II pseudomonads based on $16 \mathrm{~S}$ rRNA gene sequences. J. Appl. Bacteriol. 74:324-329

25. Lindberg, G. D. 1981. An antibiotic lethal to fungi. Plant Dis. 65:680-683.

26. Lindberg, G. D., and J. M. Larkin. 1980. Production of tropolone by Pseudomonas. J. Nat. Prod. (Lloydia) 43:592-594.

27. Oyaizu, H., and K. Komagata. 1983. Grouping of Pseudomonas species on the basis of cellular fatty acid composition and the quinone system with special reference to the existence of 3-hydroxy fatty acids. J. Gen. Appl. Microbiol. 29:17-40.

28. Palleroni, N. J. 1984. Genus 1. Pseudomonas Migula $1894,237^{\mathrm{AL}}$ (Nom. cons. Opin. 5, Jud. Comm. 1952,237), p. 141-199. In N. R. Krieg and J. G. Holt (ed.), Bergey's manual of systematic bacteriology, vol. 1. The Williams \& Wilkins Co., Baltimore.

29. Palleroni, N. J., R. W. Ballard, E. Ralston, and M. Doudoroff. 1972. Deoxyribonucleic acid homologies among some Pseudomonas species. J. Bacteriol. 110:1-11.

30. Palleroni, N. J., and M. Doudoroff. 1971. Phenotypic characterization and deoxyribonucleic acid homologies of Pseudomonas solanacearum. J. Bacteriol. 107:690-696.

31. Palleroni, N. J., and B. Holmes. 1981. Pseudomonas cepacia sp. nov., nom. rev. Int. J. Syst. Bacteriol. 31:479-481.

32. Palleroni, N. J., R. Kunisawa, R. Contopoulou, and M. Doudoroff. 1973. Nucleic acid homologies in the genus Pseudomonas. Int. J. Syst. Bacteriol. 23:333-339.

33. Pickett, M. J., and J. R. Greenwood. 1980. A study of the Va-1 group of pseudomonads and its relationship to Pseudomonas pickettii. J. Gen. Microbiol. 120:439-446.

34. Ralston, E., N. J. Palleroni, and M. Doudoroff. 1973. Pseudomonas pickettii, a new species of clinical origin related to Pseudomonas solanacearum. Int. J. Syst. Bacteriol. 23:15-19.

35. Ramundo, B. A., and L. E. Claflin. 1990. Demonstration of synonymy between the plant pathogens Pseudomonas avenae and Pseudomonas rubrilineans. J. Gen. Microbiol. 136:2029-2033.
36. Rosen, H. R. 1922. A bacterial disease of foxtail (Chaetochloa lutescens). Ann. Mo. Bot. Gard. 9:333-402.

37. Saito, H., and K. Miura. 1963. Preparation of transforming deoxyribonucleic acid by phenol treatment. Biochim. Biophys. Acta 72:619-629.

38. Schaad, N. W., C. I. Kado, and D. R. Sumner. 1975. Synonymy of Pseudomonas avenae Manns 1905 and Pseudomonas alboprecipitans Rosen 1922. Int. J. Syst. Bacteriol. 28:133-137.

39. Skerman, V. B. D., V. McGowan, and P. H. A. Sneath (ed.). 1980. Approved lists of bacterial names. Int. J. Syst. Bacteriol. 30:225420.

40. Stackebrandt, E., R. G. E. Murray, and H. G. Truper. 1988. Proteobacteria classis nov., a name for the phylogenetic taxon that includes the "purple bacteria and their relatives." Int. J. Syst. Bacteriol. 38:321-325.

41. Stahl, D. A., D. J. Lane, G. J. Olsen, D. J. Heller, T. M. Schmidt, and N. R. Pace. 1987. Phylogenetic analysis of certain sulfideoxidizing and related morphologically conspicuous bacteria by $5 \mathrm{~S}$ ribosomal ribonucleic acid sequences. Int. J. Syst. Bacteriol. 37:116-122.

42. Stanier, R. Y., N. J. Palleroni, and M. Doudoroff. 1966. The aerobic pseudomonads: a taxonomic study. J. Gen. Microbiol. 43:159-271.

43. Stead, D. E. 1992. Grouping of plant-pathogenic and some other Pseudomonas spp. by using cellular fatty acid profiles. Int. J. Syst. Bacteriol. 42:281-295.

44. Tamaoka, J., D. Ha, and K. Komagata. 1987. Reclassification of Pseudomonas acidovorans den Dooren de Jong 1926 and Pseudomonas testosteroni Marcus and Talalay 1956 as Comamonas acidovorans comb. nov. and Comamonas testosteroni comb. nov., with an emended description of the genus Comamonas. Int. J. Syst. Bacteriol. 37:52-59.

45. Tamaoka, J., and K. Komagata. 1984. Determination of DNA base composition by reversed high-performance liquid chromatography. FEMS Microbiol. Lett. 25:125-128.

46. Uematsu, T., D. Yoshimura, K. Nishiyama, T. Ibaraki, and H. Fujii. 1976. Pathogenic bacterium causing seedling rot of rice. Ann. Phytopathol. Soc. Jpn. 42:464-471.

47. Urakami, T., H. Araki, H. Oyanagi, K. Suzuki, and K. Komagata. 1990. Paracoccus aminophilus sp. nov., and Paracoccus aminovorans sp. nov., which utilize $N, N$-dimethylformamide. Int. J. Syst. Bacteriol. 40:287-291.

48. Urakami, T., and K. Komagata. 1979. Cellular fatty acid composition and coenzyme $Q$ system in gram-negative methanol-utilizing bacteria. J. Gen. Appl. Microbiol. 25:343-360.

49. Urakami, T., and K. Komagata. 1986. Occurrence of isoprenoid compounds in gram-negative methanol-, methane-, and methylamine-utilizing bacteria. J. Gen. Appl. Microbiol. 32:317-341.

50. Urakami, T., and K. Komagata. 1987. Cellular fatty acid composition with special reference to the existence of hydroxy fatty acids in gram negative methanol-, methane-, and methylamine-utilizing bacteria. J. Gen. Appl. Microbiol. 33:135-165.

51. Wakimoto, S., K. Hirayae, K. Tsuchiya, Y. Kushima, N. Furuya, and N. Matsuyama. 1986. Production of antibiotics by plant pathogenic Pseudomonas. Ann. Phytopathol. Soc. Jpn. 52:835-842.

52. Willems, A., J. Busse, M. Goor, B. Pot, E. Falsen, E. Jantzen, B. Hoste, M. Gillis, K. Kersters, G. Auling, and J. De Ley. 1989. Hydrogenophaga, a new genus of hydrogen-oxidizing bacteria that includes Hydrogenophaga flava comb. nov. (formerly Pseudomonas flava), Hydrogenophaga palleronii (formerly Pseudomonas palleronii), Hydrogenophaga pseudoflava (formerly Pseudomonas pseudoflava and "Pseudomonas carboxydoflava"), and Hydrogenophaga taeniospiralis (formerly Pseudomonas taeniospiralis). Int. J. Syst. Bacteriol. 39:319-333.

53. Willems, A., E. Falsen, B. Pot, E. Jantzen, B. Hoste, P. Vandamme, M. Gillis, K. Kersters, and J. De Ley. 1990. Acidovorax, a new geus for Pseudomonas facilis, Pseudomonas delafieldii, EF group 13, EF group 16, and several clinical isolates, with the species Acidovorax facilis comb. nov., Acidovorax delafieldii comb. nov., and $A C$ idovorax temperans sp. nov. Int. J. Syst. Bacteriol. 40:384-398.

54. Willems, A., M. Goor, S. Thielemans, M. Gillis, K. Kersters, and J. De Ley. 1992. Transfer of several phytopathogenic Pseudomonas species to Acidovorax as Acidovorax avenae subsp. avenae subsp. 
nov., comb. nov., Acidovorax avenae subsp. citrulli, Acidovorax avenae subsp. cattleyae, and Acidovorax konjaci. Int. J. Syst. Bacteriol. 42:107-119.

55. Willems, A., B. Pot, E. Falsen, P. Vandamme, M. Gillis, K. Kersters, and J. De Ley. 1991. Polyphasic taxonomic study of the emended genus Comamonas: relationship to Aquaspirillum aquaticum, E. Falsen group 10, and other clinical isolates. Int. J. Syst. Bacteriol. 41:427-444.

56. Woese, C. R. 1987. Bacterial evolution. Microbiol. Rev. 51:221271.

57. Woese, C. R., P. Blanz, and C. M. Hahn. 1984. What isn't a pseudomonad: the importance of nomenclature in bacterial classification. Syst. Appl. Microbiol. 5:179-195.

58. Woese, C. R., W. G. Weisburg, C. M. Hahn, B. J. Paster, L. B. Zablen, B. J. Lewis, T. J. Macke, W. Ludwig, and E. Stackebrandt. 1985. The phylogeny of purple bacteria: the gamma subdivision. Syst. Appl. Microbiol. 6:25-33.
59. Woese, C. R., W. G. Weisburg, B. J. Paster, C. M. Hahn, R. S. Tanner, N. R. Krieg, H. P. Koops, H. Harms, and E. Stackebrandt. 1984. The phylogeny of purple bacteria: the beta subdivision. Syst. Appl. Microbiol. 5:327-336.

60. Yabuuchi, E., Y. Kosako, H. Oyaizu, I. Yano, H. Hotta, Y. Hashimoto, T. Ezaki, and M. Arakawa. 1992. Proposal of Burkholderia gen. nov. and transfer of seven species of the genus Pseudomonas homology group II to the new genus, with the type species Burkholderia cepacia (Palleroni and Holmes 1981) comb. nov. Microbiol. Immunol. 36:1251-1275.

61. Yamada, Y., H. Takinami-Nakamura, Y. Tahara, H. Oyaizu, and K. Komagata. 1982. The ubiquinone systems in the strains of Pseudomonas species. J. Gen. Appl. Microbiol. 28:7-12.

62. Young, J. M., D. W. Dye, J. F. Bradbury, C. G. Panagopoulos, and C. F. Robbs. 1978. A proposed nomenclature and classification for plant pathogenic bacteria. N. Z. J. Agric. Res. 21:153-177. 